EPJ Web of Conferences 59, 02011 (2013)

DOI: $10.1051 /$ epjconf/20135902011

(C) Owned by the authors, published by EDP Sciences, 2013

\title{
NIF capsule performance modeling
}

\author{
S. Weber ${ }^{1}$, D. Callahan ${ }^{1}$, C. Cerjan ${ }^{1}$, M. Edwards ${ }^{1}$, S. Haan ${ }^{1}$, D. Hicks ${ }^{1}$, \\ O. Jones ${ }^{1}$, G. Kyrala ${ }^{2}$, N. Meezan ${ }^{1}$, R. Olson ${ }^{3}$, H. Robey ${ }^{1}$, B. Spears ${ }^{1}$, \\ P. Springer ${ }^{1}$ and R. Town ${ }^{1}$
}

${ }^{1}$ Lawrence Livermore National Laboratory, Livermore, CA 94550, USA

${ }^{2}$ Los Alamos National Laboratory. Los Alamos, NM 87545, USA

${ }^{3}$ Sandia National Laboratory, Albuquerque, NM 87185, USA

\begin{abstract}
Post-shot modeling of NIF capsule implosions was performed in order to validate our physical and numerical models. Cryogenic layered target implosions and experiments with surrogate targets produce an abundance of capsule performance data including implosion velocity, remaining ablator mass, times of peak x-ray and neutron emission, core image size, core symmetry, neutron yield, and x-ray spectra. We have attempted to match the integrated data set with capsule-only simulations by adjusting the drive and other physics parameters within expected uncertainties. The simulations include interface roughness, timedependent symmetry, and a model of mix. We were able to match many of the measured performance parameters for a selection of shots.
\end{abstract}

National Ignition Facility (NIF) ignition target must satisfy stringent criteria in order to achieve the conditions for ignition [1]. These include the timing of shocks through the DT ice layer, implosion velocity, time-dependent drive symmetry, and x-ray and hot electron preheat limits. Surrogate targets are used to tune the target and laser parameters to within the requirements. Reemit balls are use to tune the drive symmetry during the first "picket" of the laser pulse [2]. "Keyhole" targets are used to time the shocks through the DT ice layer [3, 4]. Symmetry capsules (SymCaps) are gas-filled shells fielded to assess the radiation drive symmetry by observing the shape of the x-ray emission from the stagnating core [5]. Convergent ablation experiments also employ gas-filled capsules, but are back-lit inflight to measure the implosion velocity and the ablator mass remaining at the time of peak velocity $[6,7]$. Finally, THD capsules have cryogenic fuel layers depleted in deuterium to reduce reactivity [8]. Lower yield facilitates diagnosing whether the conditions required for ignition have been achieved.

The NIF diagnostic suite provides a wealth of data for THD or DT cryogenic-layered implosions and for the surrogate target experiments employed in tuning. From convergent ablation experiments we have shell center of mass radius vs. time, shell velocity, $\rho \Delta$ r, unablated shell mass, shell thickness, and peak density. X-ray imaging of the compressed core of SymCap and THD capsules gives core radius, symmetry, brightness, and spectral information from a crystal spectrometer and pass-band filters. Neutron diagnostics give neutron yield, burn-weighted ion temperature, and down-scatter ratio "dsr", which is the ratio of the number of $10-12 \mathrm{MeV}$ neutrons to $13-15 \mathrm{MeV}$ neutrons. The $10-12 \mathrm{MeV}$ neutrons are produced mostly by scattering from hydrogen isotopes so the ratio is proportional to burnaveraged fuel $\rho \Delta \mathrm{r}$.

Post-shot modeling on NIF capsule implosions has been carried out to validate physical and numerical models. Ideally, a simulation starting with the detailed metrology of the actual target employed for a specific laser shot and the measured incident power for each of the 192 NIF beams would reproduce the diagnostic measurements. In practice, the physics models used in the simulations are not accurate enough to match a specific shot, which is why empirical tuning is needed. Additionally,

This is an Open Access article distributed under the terms of the Creative Commons Attribution License 2.0, which permits unrestricted use, distribution, and reproduction in any medium, provided the original work is properly cited. 

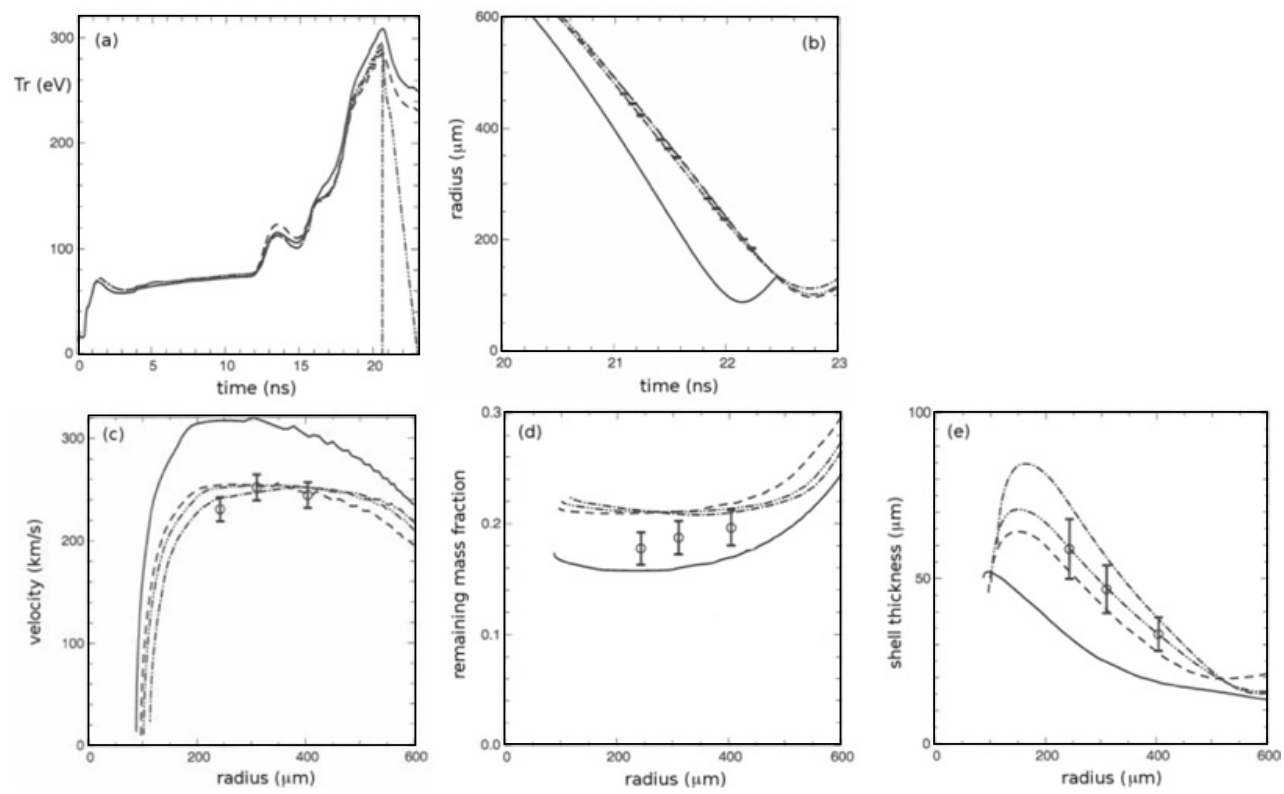

Figure 1. (a) Radiation drive $\operatorname{Tr}(\mathrm{t})$ options for convergent ablation shot $\mathrm{N} 110625$, solid-full integrated simulation drive, dashed-drive reduced $20 \%$ in the $4^{\text {th }}$ pulse, dashdot-reduced and truncated after the peak, dashdotdot-rapid fall; (b) radius vs. time, data show as error bars, line styles as before; (c) velocity vs. radius; (d) remaining mass fraction vs. radius; (e) shell thickness 2 xstandard deviation vs. radius.

processes may lie outside of expected uncertainties or be missing altogether in our model, which could lead to results outside of the predicted tuning range and frustrate tuning.

In this paper we describe efforts to match diagnostic results from recent implosions of NIF Ge-doped $\mathrm{CH}$ layered capsules. We are able to match many observables with simulations tuned to reproduce the symmetry, shock-timing, and velocity history of surrogate targets. The drive flux was reduced from predictions of integrated hohlraum simulations to match the implosion trajectory [6, 7]. Simulations with this drive match with decreasing fidelity SymCaps, layered target implosions with drive for which the shocks were mis-timed, and layered target implosions with shock-timed drive.

The simulations described here are of the capsule only, using an applied time- and spectrallydependent radiation source. Time-dependent drive asymmetry is included as spherical harmonic $\mathrm{Y}_{l m}$ modes up to $1=6$. As compared to integrated simulations including the hohlraum, this allows use of higher spatial resolution. Most of these simulations are two-dimensional, including 512 zones in $90^{\circ}$, allowing resolution of ablator and ice surface finish modes up to $l=100$. With this methodology it is simpler to adjust the drive to match the measured implosion trajectory, radius vs. time.

The drive up to the rise of the $4^{\text {th }}$ pulse was adjusted to match shock timing data [9]. The drive after the rise of the $4^{\text {th }}$ pulse is not constrained by the keyhole shots, but rather by the convergent ablation shots. Simulations using drive calculated with the LASNEX high flux model give an implosion trajectory which is too fast and early compared to data. Fits may be obtained by lowering the peak flux by $\sim 20 \%$ (see Fig. 1). The fit is not unique. A drive which is truncated abruptly after the peak gives a better match to the shell velocity vs. time (Fig. 1c). A drive which falls $4 \mathrm{x}$ faster than simulated does not match as well as the truncated case.

The convergent ablation experiments also measure the mass remaining in the shell and the in-flight shell thickness. The full drive case matches the remaining mass while none of the reduced drive cases do (Fig. 1d). The rapidly-dropping drive option gives a good match to the in-flight shell thickness (Fig. 1e). 

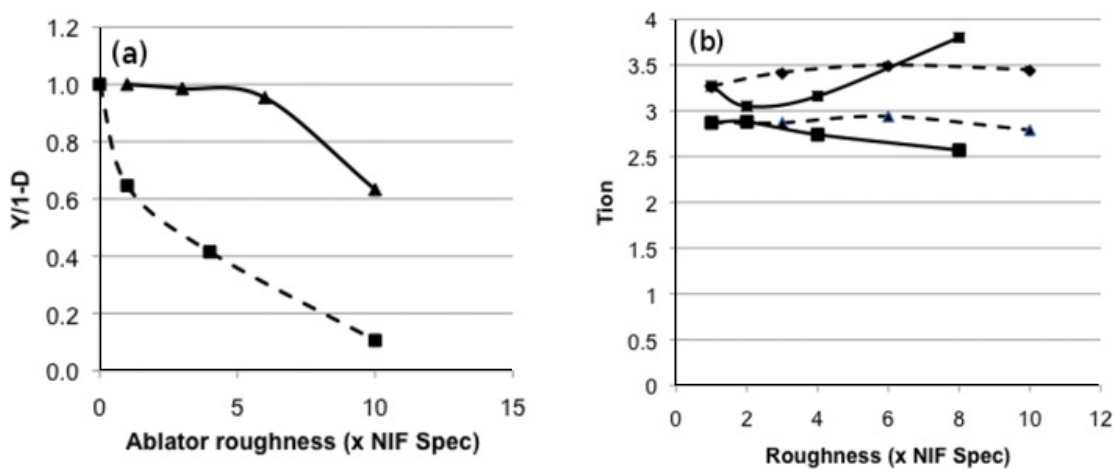

Figure 2. (a) Yield divided by 1-D, solid-not shock-timed drive of shot N110212, dash-shock-timed drive of N110603; (b) Ion temperature vs. roughness as a multiplier over the NIF specification, solid-ice roughness, dashablator roughness, lower pair of curves-kinetic Tion, upper pair-Tion fit to escaped neutron spectrum.

In February, 2011 several SymCaps and a THD layered implosion were carried out with a drive with mis-timed shocks. These were scoping experiments before careful shock timing, first performed in May, 2011. These implosions gave symmetric images. Simulations with drive matched to the convergent ablation experiments reproduce SymCap results well (Table 1). A convergent ablation experiment was not done with the actual $1.3 \mathrm{MJ}$ drive of these shots. We used a drive with multipliers inferred from a $1 \mathrm{MJ}$ convergent ablation shot in December. This drive matches the measured bang time for the $1.3 \mathrm{MJ}$ shots. All simulations included $\alpha$-particle deposition.

The layered target shot in February gave about half of preshot calculated yield. Our post-shot simulations with the adjusted drive model reproduce many of the measured data. The simulated yield is larger than measured. Two-dimensional simulations using measured ice and ablator roughness still predict too high yield. The amount of mix may be raised by supposing that the ice or ablator roughness is larger than measured, or equivalently that the instability growth is larger than calculated. Performance degrades only slowly as the assumed roughness is increased. We have simulated ablator roughness enhanced by up to 10x and ice roughness up to $8 \mathrm{x}$ (Fig. 2). Either of these enhancements reduces the predicted yield by not quite the required factor two.

One would expect that mix would lower the ion temperature, while the measured ion temperature is often larger than simulations predict. The ion temperature is inferred from Doppler broadening of the $14 \mathrm{MeV}$ neutron peak. Kinetic temperature broadens the peak. However, bulk motion of fuel also contributes to the broadening of the peak. Roughness increases internal motions, so we find that the broadening of the $14 \mathrm{MeV}$ feature increases even as the kinetic temperature of the fuel drops. Fig. 2 shows how the ion temperature inferred from synthetic neutron spectra increases with roughness, especially of the ice, while the kinetic temperature drops as expected.

Several targets with THD or DT ice layers were imploded during June, 2011 with a drive for which the shocks had been timed with keyhole shots performed in May [3, 4]. We found that after the shock timing we were not to recover implosion symmetry by adjusting the wavelength difference between the inner and outer cone beams. In addition, significant non-axisymmetric asymmetry was very apparent in the polar $\mathrm{x}$-ray images, especially large amplitude of spherical harmonic $\mathrm{m}=4$. However, the dsr did increase from that of the February shot, to $\sim 0.045$. For these shots, the neutron yield dropped to $\sim 0.05$ of that predicted by preshot simulations. We have selected the N110603 THD implosion as representative of this set of layered shots to test our model (See Table 2; radius is the $1=0$ coefficient of a Legendre polynomial fit to the $17 \% \mathrm{x}$-ray contour at peak brightness).

Three-dimensional simulations to examine the effect of the $\mathrm{m}=4$ structure seen in the $\mathrm{x}$-ray images lowered the predicted yield by only a factor of three compared to a 1-D simulation with the same drive. In 
EPJ Web of Conferences

Table 1. Performance metrics of February, 2011 implosions which did not have timed shocks.

\begin{tabular}{|l|c|c|c|c|c|c|c|}
\hline type & $\begin{array}{c}\text { N110208 } \\
\text { SymCap }\end{array}$ & 1-D sim & 2-D sim & $\begin{array}{c}\text { N110212 } \\
\text { THD }\end{array}$ & 1-D sim & 2-D sim & $\begin{array}{c}\text { 2-D sim } \\
\text { roughx10 }\end{array}$ \\
\hline BT $(\mathbf{n s})$ & 21.34 & 21.21 & 21.15 & 21.3 & 21.28 & 21.29 & 21.20 \\
\hline Yn & $8.00 \mathrm{e} 11$ & $8.79 \mathrm{e} 11$ & $6.85 \mathrm{e} 11$ & $1.30 \mathrm{e} 14$ & $3.04 \mathrm{e} 14$ & $2.86 \mathrm{e} 14$ & $1.96 \mathrm{e} 14$ \\
\hline Ti $(\mathbf{k e V})$ & 3.15 & 2.83 & 2.71 & 3.7 & 2.76 & 3.33 & 3.45 \\
\hline dsr & & & & 0.025 & 0.0384 & 0.0357 & 0.0302 \\
\hline radius $(\mu \mathbf{m})$ & 62 & 53.7 & 53.0 & 42 & 31.6 & 35.7 & 36.4 \\
\hline
\end{tabular}

Table 2. Performance metrics of June, 2011 implosions which had timed shocks.

\begin{tabular}{|l|c|c|c|c|c|c|}
\hline type & N110603 THD & 1-D & 1-D & 2-D & 2-D rough x 4 & 3-D \\
\hline rapid fall drive & & no & yes & yes & yes & no \\
\hline BT $(\mathbf{n s})$ & $22.3( \pm 0.1)$ & 22.41 & 22.48 & 22.48 & 22.35 & 22.35 \\
\hline Yn & $6.5( \pm 0.1) \mathrm{e} 13$ & $1.45 \mathrm{e} 15$ & $1.76 \mathrm{e} 14$ & $1.38 \mathrm{e} 14$ & $7.30 \mathrm{e} 13$ & $4.52 \mathrm{e} 14$ \\
\hline Ti $(\mathbf{k e V})$ & $2.6 \pm 0.2$ & 2.81 & 2.43 & 2.81 & 3.16 & 3.05 \\
\hline dsr & $0.044 \pm 0.003$ & 0.0576 & 0.049 & 0.042 & 0.040 & 0.0448 \\
\hline radius $(\mu \mathbf{m})$ & $33 \pm 2$ & 24.9 & 28.6 & 26.8 & 24.1 & 20.5 \\
\hline x-ray $\mathbf{P}_{\mathbf{2}} / \mathbf{P}_{\mathbf{0}}$ & $-59 \%$ & & & $-28 \%$ & -0.24 & $-25 \%$ \\
\hline $\mathbf{x}-\mathbf{r a y} \mathbf{m}_{\mathbf{4}} / \mathbf{m}_{\mathbf{0}}$ & $6.8 \%$ & & & & & $10.2 \%$ \\
\hline
\end{tabular}

contrast, the rapidly-falling drive in Fig. 1a lowered the predicted yield by nearly an order of magnitude compared to the drive reduced by a constant factor. Implosions with shock-timed drive degraded more rapidly with increasing ablator roughness, compared to the un-timed drive (Fig. 2) because shock timing makes the shell thinner inflight. Two-dimensional simulations with the rapidly-falling drive and $4 \mathrm{x}$ specification ablator roughness match the yield of the N110603 shot. This simulation is in fair agreement with the dsr, ion temperature, and burn duration of the shot. The x-ray image size is still too small by $23 \%$. Further experiments are needed to test whether the effective drive for NIF capsules falls off after the peak as rapidly as this model supposes. Dante data is consistent with nominal drive modeling, but the laser entrance hole closes very rapidly after the laser is off making the internal radiation temperature uncertain.

In conclusion, simulations tuned to reproduce the symmetry, shock-timing, and velocity history measured in surrogate target implosions are able to match many of the observables of layered target implosions. A better match to the observed yield is obtained by assuming higher ablator or ice roughness than is inferred from target metrology.

\section{References}

[1] O. Landen et al., Phys Plasmas 18, 051002 (2011)

[2] E. Dewald et al., Phys Plasmas 18, 092703 (2011)

[3] P. Celliers et al., these proceedings (2011)

[4] H. Robey et al., these proceedings (2011)

[5] G. Kyrala et al., Phys Plasmas 18, 056307 (2011)

[6] D. Hicks et al., Phys Plasmas 17, 102703 (2010)

[7] R. Olson et al., these proceedings (2011)

[8] J. Edwards et al., Phys Plasmas 18, 051003 (2011)

[9] O. Jones et al., these proceedings (2011) 